\title{
Cortical excitability and the shape of the haemodynamic response
}

Haigh S. M. ${ }^{1 \text { a }}$, Cooper N.R. ${ }^{2} \&$ Wilkins A. J. ${ }^{3}$

Department of Psychology, University of Essex, Wivenhoe Park, Colchester, Essex, CO4 3SQ, UK.

${ }^{1}$ haighsm@upmc.edu; ${ }^{2}$ ncooper@essex.ac.uk; ${ }^{3}$ arnold@essex.ac.uk

Present address for correspondence:

Sarah M. Haigh,

Clinical Neurophysiology Research Laboratory,

Department of Psychiatry, School of Medicine,

University of Pittsburgh and UPMC,

Suite 420 Oxford Building,

3501 Forbes Avenue,

Pittsburgh, PA 15212.

(412) 586-9073.

Email: haighsm@upmc.edu

${ }^{a}$ Author SMH has since moved to University of Pittsburgh, CNRL, Oxford Building Suite 420, 3501 Forbes Avenue, Pittsburgh, PA, 15213, USA. 


\section{Abstract}

Individual differences in the temporal dynamics of the haemodynamic response can reflect cortical excitation and can reveal underlying cortical physiology. Here, we show differences in the shape of the haemodynamic response that are dependent on stimulus parameters. Two sets of visual stimuli were used varying in parameters that are known to manipulate the haemodynamic response in the visual cortex. We measured the oxyhaemoglobin response using near infrared spectroscopy. The first set of stimuli comprised chromatic square-wave gratings that varied with respect to the separation in the $\mathrm{CIE}$ UCS chromaticities of the alternating bars. The gratings with large separations in chromaticity evoked an oxyhaemoglobin response with greater amplitude, consistent with greater activation of the visual cortex. The second set of stimuli comprised horizontal achromatic gratings that (1) were static, (2) drifted at a constant velocity towards fixation, or (3) reversed direction every half spatial cycle to create a vertical vibrating motion. Although the three types of grating had a similar effect on the amplitude of the oxyhaemoglobin response, the moving gratings ( 2 and 3 ) evoked a steeper decrease in oxyhaemoglobin concentration after stimulus-offset. The steeper slope appears to reflect the poststimulus undershoot and the slope may provide a correlate of cortical excitability when the amplitude of the haemodynamic response has saturated.

Keywords: NIRS, oxyhaemoglobin, excitability, haemodynamic response 


\section{Introduction}

Near-infrared spectroscopy (NIRS) uses light to measure changes in spectral reflectance of oxygenated and deoxygenated blood in the cortex (Villringer et al., 1993). The temporal resolution of NIRS is higher than that of fMRI (although its spatial resolution is much poorer) and NIRS offers the opportunity for closer interrogation of the temporal properties of the haemodynamic response.

The relationship between haemodynamic changes and neural excitation is complicated but is related to local field potentials (Logothetis, 2001). The haemodynamic response can therefore be used as a surrogate measure of neural activation. Comparisons of $\mathrm{fMRI} / \mathrm{NIRS}$ responses to visual stimuli and findings from electroencephalography (EEG) studies to the same stimuli explicitly test the relationship between the haemodynamic and the neural response. Visual stimuli that evoke a strong neural response also produce a large change in the haemodynamic response in visual cortex. For example, the fMRI BOLD signal increases linearly with log contrast (Olman, Ugurbil, Schrater \& Kersten, 2004; Vazquez \& Noll, 1998), and high log contrast produces greater oxyhaemoglobin responses measured using NIRS (Rovati, Salvatori, Bulf \& Fonda, 2007). Higher contrast grating patterns also increase the amplitude of visual evoked potentials and increase gamma activity in visual cortex (Schadow et al., 2007). However, there is some debate over the sensitivity of the haemodynamic response to changes in spatial frequency (compare Swettenham et al., 2013, with Huang et al., 2003). Tong et al. (2005) measured NIRS and EEG responses to an auditory stimulus in an odd-ball paradigm, and found an increase in oxyhaemoglobin a few seconds after the presence of a P300 component in an ERP response. Simultaneous NIRS and EEG recordings have also been used to measure the relationship between haemodynamic responses and alpha activity (Moosmann et al., 2003) and epileptogenic activity (for example, see Gallagher et al., 2008; Lareau et al., 2011; Roche-Labarbe et al., 2008).

There are several independent indications of a cortical hyperexcitability in migraine (see Aurora \& Wilkinson, 2007 for a review). For example, Huang et al. (2003) found that patients with migraine showed larger BOLD responses to grating stimuli than controls. It is therefore of interest that Coutts et al. (2012) using NIRS found that patients with migraine showed shorter oxyhaemoglobin responses compared to healthy controls, suggesting that the shape of the haemodynamic response can also be related to cortical excitability. This suggestion is consistent with the findings of Muthukumaraswamy et al. (2012) that individuals with lower concentrations of GABA produced taller and thinner BOLD responses in the visual cortex, compared to individuals with higher concentrations of GABA. GABAergic mechanisms affect local cortical excitability (Semyanov et al., 2003). It is therefore possible that the 
shape of the haemodynamic response, as well as its amplitude, can reflect cortical excitability. Consequently we wanted to test the relationship between the amplitude and shape of the oxyhaemoglobin response to different visual stimuli.

Here we present two experiments in which we measured the relationship between the amplitude and the shape of the oxyhaemoglobin response using NIRS. We manipulated stimulus parameters that have been shown to affect viewing comfort and to influence the probability of paroxysmal EEG activity in patients with photosensitive epilepsy. Following the findings reported by Muthukumaraswamy et al. (2012) and Coutts et al. (2012), we chose to focus on the effect of the stimulus parameters on the oxyhaemoglobin response during stimulus presentation and immediately after stimulus presentation, and the effect on the size of the post-stimulus undershoot (PSU). Our aim was to measure the haemodynamic response in relation to cortical excitation.

In the first experiment, we presented isoluminant chromatic square-wave gratings that varied in the separation between the chromaticities of the component bars. Gratings with large chromaticity separation are uncomfortable to view and can produce a large haemodynamic response (Haigh et al., 2013). Flicker in which two coloured lights alternate can evoke paroxysmal EEG activity in patients with photosensitive epilepsy (Parra et al., 2007), and the probability of paroxysmal activity increases with the separation in chromaticity. For the second experiment, we presented achromatic square-wave gratings that were (1) static (2) drifted at a constant $(10 \mathrm{~Hz})$ velocity, or (3) drifted at the same velocity but reversed direction every half spatial cycle causing the stripes to vibrate. $10 \mathrm{~Hz}$ vibrating frequency was associated with the highest probability of a photoparoxysmal response in patients with photosensitive epilepsy (Binnie et al., 1985). Achromatic gratings with a drifting or vibrating motion are particularly uncomfortable to view, when compared to a static grating (Haigh et al., 2012), and the vibrating motion is epileptogenic (Binnie et al., 1985). These stimuli vary in their 'strength' which should offer a range of cortical stimulation sufficient to measure any relationship between the amplitude and the shape of haemodynamic responses.

\section{General Methods}

\subsection{Participants}

Nineteen females and three males from the University of Essex participated in the study; mean age 21.2 (range 18-54). All had a minimum of 6/6 visual acuity (Lighthouse Test for Near and Far Visual Acuity), a minimum stereo acuity of 60 secs.arc (Titmus test; Stereo Optical Co. In., Chicago, IL, USA), and showed 
no red-green colour deficiencies (Ishihara plates). This study complied with the Code of Ethics of the World Medical Association (Declaration of Helsinki), and was approved by the University of Essex Review Board. All participants gave their informed consent.

\subsection{Apparatus}

The stimuli were displayed on a 24" LCD screen (Dell corporation model 2408) with a backlight modulation frequency of $162.5 \mathrm{~Hz}$. The Michelson contrast of the modulation was 0.86 . For the chromatic gratings, the screen was powered by a Mac G4 Powerbook. For the moving gratings, the screen was powered by a Dell Precision M4500 laptop. A photo diode and associated electronics provided a trigger for the NIRS system.

\subsection{Stimuli for Experiment 1 - Static Chromatic Gratings}

Gratings were displayed using SuperLab version 4.0.7b. To select the chromaticities, the three extremes of the screen gamut (red pixels only, green only and blue only) were measured using a telespectroradiometer (model PR-670 ${ }^{\circledR}$, Photo Research ${ }^{\circledR}$, Chatsworth, CA, USA). The isoluminant midpoints $\left(29 \mathrm{~cd} / \mathrm{m}^{2}\right)$ between each of the three extremes were calculated. Chromaticities equidistant from either side of the midpoint were then used in alternating stripes to create red-green (RG), greenblue (GB) and red-blue (RB) grating patterns. The spatial frequency of the patterns was $2 \mathrm{cpd}$ at a viewing distance of $1 \mathrm{~m}$. Three color pairs were created for each gamut extreme: a small separation of chromaticities in the CIE UCS diagram (mean separation of the chromaticities $=0.03$ ), a medium separation (mean separation of the chromaticities $=0.19$ ) and a large separation (mean separation of the chromaticities $=0.43$ ). The gratings were circular in outline, subtended $10 \mathrm{deg}$, and were surrounded by a grey field of similar luminance. A central black fixation cross was shown throughout the trial (subtending 1.3 degrees of visual angle). The order of the stimuli was randomized and each pattern was presented twice.

\subsection{Stimuli for Experiment 2 - Static and Moving Achromatic Gratings}

Horizontal gratings were created and presented in MATLAB ${ }^{\circledR}$ using the PsychToolbox extension

(Brainard, 1997; Pelli, 1997; Kleiner et al., 2007). All the gratings were achromatic (CCT $4000^{\circ} \mathrm{K}$ ) and had a square-wave luminance profile. The contrast of the gratings was vignetted with a Gaussian filter to reduce edge effects. At half-contrast the gratings subtended 9.1 degrees of visual angle. The gratings had a spatial frequency of $2 \mathrm{cpd}$. Vibrating and drifting gratings moved vertically with a contour velocity of 20 degrees/s $(10 \mathrm{~Hz})$, and to prevent optokinetic nystagmus, they moved symmetrically in opposite directions either side of a horizontal midline (upwards in the upper field and downwards in the lower). 
The profile of motion for the vibrating grating was a triangle wave: the grating drifted vertically at a constant velocity either up or down through one half spatial cycle before abruptly reversing direction. The stimuli were presented in the following order: drifting, vibrating, static, static, vibrating, drifting, static, drifting, vibrating, vibrating, drifting, static, vibrating, static, drifting, static, vibrating, drifting.

\subsection{Procedure}

The haemodynamic response was measured using a 10 channel split-receiver near infrared spectroscopy (NIRS) system (Oxymon Mk II Artinis Medical Systems BV Zetten, Netherlands). The optode placement is shown in Figure 1. Two receivers were placed symmetrically $20 \mathrm{~mm}$ above the inion and $30 \mathrm{~mm}$ either side of the midline. Two transmitters were then placed vertically $30 \mathrm{~mm}$ from the receiver, covering 01 and $\mathrm{O} 2$ (according to the 10-20 system for electrode placement). Four more transmitters were then placed laterally around each of the receivers at $40^{\circ}$ intervals, with a radius of $30 \mathrm{~mm}$ from the receiver. Two additional transmitters and receivers were placed over F1 and F2 to check that the responses to the stimuli were purely visual and were not systemic. In the event, the signal from these channels was generally very low.

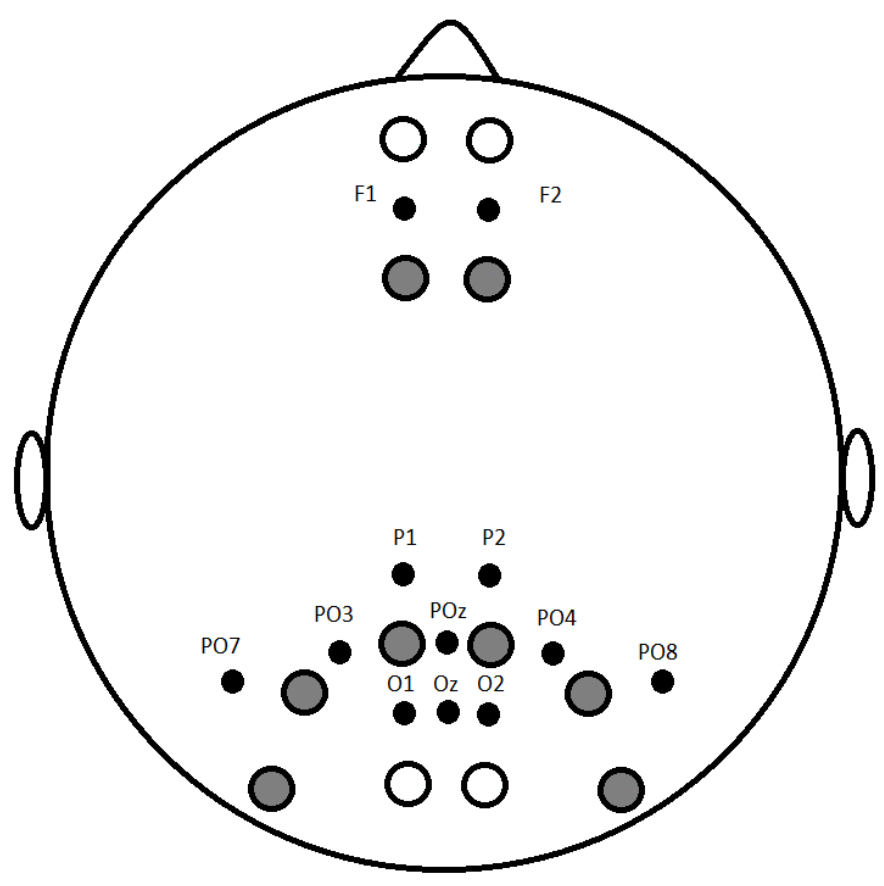

Figure 1. The layout of the NIRS optodes. The grey circles show the NIRS transmitters, the white circles the NIRS receivers, and the black circles show the 10-20 EEG electrode locations that were used as a guide for optode placement. 
Each stimulus was presented for 16 s to allow the haemodynamic response to reach its maximum, and was then followed by a grey screen to allow the haemodynamic response to return to baseline. The grey screen that separated each stimulus presentation lasted for an interval that varied randomly between 27-36s with a uniform probability distribution. The participants viewed the stimuli binocularly in a darkened room and were asked to fixate on a cross displayed at the centre of the screen throughout the trial.

For half the participants, the chromatic gratings were shown first followed by the achromatic gratings and the order was reversed for the remainder.

\subsection{Data Analysis}

A differential path length factor of 6.26 was assumed for the calculation of oxygenated haemoglobin concentrations in $\mu$ molar units (Duncan et al., 1996). The data were analyzed using MATLAB (The MathWorks Inc., Natick, MA), using the Signal Processing Toolbox ${ }^{\mathrm{TM}}$. The near infrared signal was filtered using a low-pass $5^{\text {th }}$ order Butterworth filter with a cut off frequency of $0.667 \mathrm{~Hz}$ to remove cardiac artefact.

Two criteria were used for excluding channels from analysis. Criterion 1: channels were excluded if they were returning an unvarying signal for more than $5 \%$ of the recording time (suggesting that the signal was obscured by hair). This resulted in 6 posterior channels being excluded (5\% loss) and 6 anterior channels being excluded from analysis ( $14 \%$ loss). The remaining channels were then grouped according to whether they covered the visual cortex (posterior channels) or the prefrontal cortex (anterior channels).

Criterion 2: In order to obtain an independent judgment as to which channels were responsive, first, the change in oxyhaemoglobin response when the stimulus was on was calculated and averaged across the stimuli, and second, the standard deviation of the signal 10s before stimulus onset was measured. If the ratio of the response change to the baseline's standard deviation was less than 1 , then the channel was rejected from further analysis.

Following the criterion, for the chromatic gratings, an average of 3.8 posterior channels from 16 participants were accepted for analysis, and were averaged separately for each participant and stimulus. Only two participants had anterior channels that passed the criterion. For the moving gratings, an average of 4.2 posterior channels from 18 participants (which included all of the channels used for the analysis of the chromatic gratings) were accepted for analysis. Only seven participants had anterior 
channels that passed the criterion. Due to the low numbers of anterior channels passing the criterion, the effects of the gratings on the anterior channels will not be reported. Note that near infrared spectroscopy has poor spatial resolution, and in consequence the functioning posterior channels were highly correlated.

Signal drift was removed from the data by fitting a linear regression over the duration of the experiment by least squares and subtracting the slope from the data. The timecourse of the oxyhaemoglobin signal was epoched around each stimulus presentation: from $27 \mathrm{~s}$ before stimulus-onset to $43 \mathrm{~s}$ after stimulusonset. The data were then smoothed with a sliding average of $2 \mathrm{~s}$. To measure the response to the grating, the baseline response (an average of the response before stimulus onset) was subtracted from the response (during stimulus presentation). The response during the stimulus was calculated from the response $6 \mathrm{~s}$ after stimulus onset for a period of $10 \mathrm{~s}$. The baseline was calculated from the response $10 \mathrm{~s}$ before stimulus onset for a period of 10s. In addition, the amplitude of the post-stimulus undershoot (PSU) of the oxyhaemoglobin response after stimulus-offset was measured: the trough of the PSU was subtracted from the baseline before stimulus-onset. The time at which the trough occurred differed for the moving gratings and the chromatic gratings (see Figure 2 to compare responses to the chromatic and moving gratings). For the response to the chromatic gratings, the PSU was calculated from the average response $22 \mathrm{~s}$ after stimulus-onset for a period of $10 \mathrm{~s}$, and the baseline was calculated from the average $14 \mathrm{~s}$ before stimulus-onset for $10 \mathrm{~s}$. For the response to the moving gratings, the PSU was calculated from the response 20 s after stimulus-onset, using the same baseline 14 s before stimulusonset.

To assess any effect of the pattern on the shape of the oxyhaemoglobin response, the slope of the rise of the response at stimulus onset, and the slope of the fall of the response at stimulus offset were measured and compared across stimulus conditions. The slopes were measured by taking the smoothed epochs and subtracting the running average signal over 6 s from the running average signal for the preceding $6 \mathrm{~s}$. The maximum difference during stimulus onset was used as the measure of the rising slope, and the minimum difference after stimulus offset was used as the measure of the falling slope.

Violations in the assumption of sphericity were corrected by adjusting the degrees of freedom using Greenhouse Geisser Correction. 


\section{Results}

\subsection{Experiment 1}

The average oxyhaemoglobin response for all the chromatic gratings is shown in Figure $2 \mathrm{~A}$, and the average oxyhaemoglobin response to all the moving gratings is shown in Figure $2 \mathrm{~B}$. The average deoxyhaemoglobin responses for the chromatic gratings and for the moving gratings are shown in Figure $2 \mathrm{C}$ and $2 \mathrm{D}$ respectively. The deoxyhaemoglobin responses were negatively correlated with the oxyhaemoglobin response $(r<-.92)$, but, possibly due to the generally smaller response, deoxyhaemoglobin responses to the different gratings were not significantly different, and will not be discussed further. 
(A)

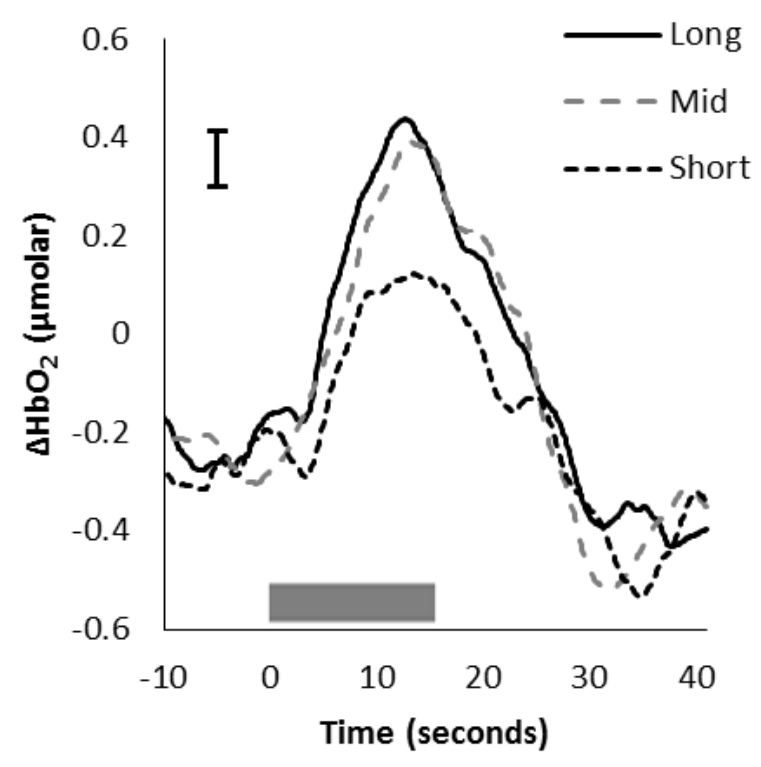

(C)

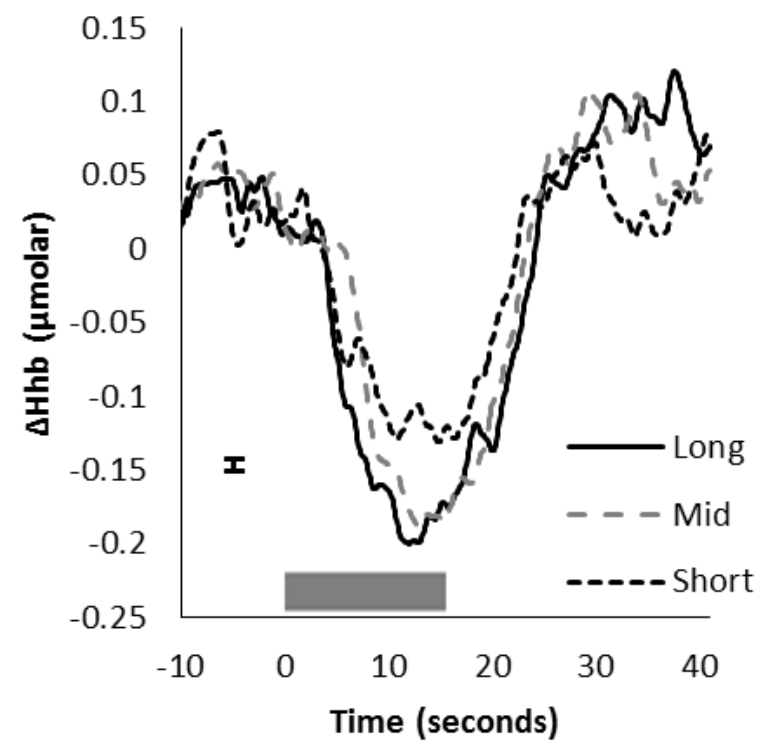

(B)

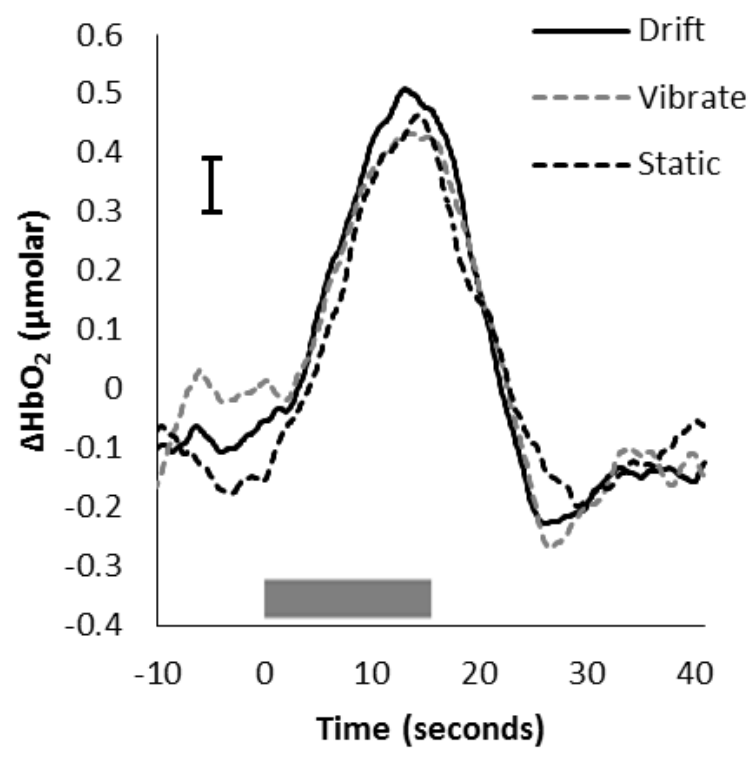

(D)

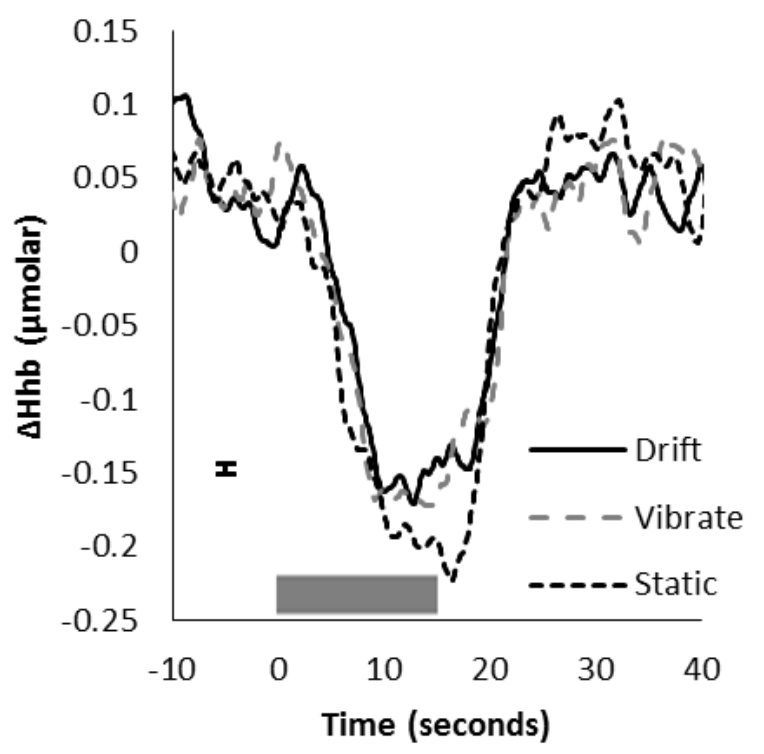

Figure 2. Top row: the average oxyhaemoglobin responses to $(A)$ the chromatic gratings, and $(B)$ the moving gratings. The vertical bars (top left) show the mean standard error (calculated over 1s) for the data in the figure. Bottom row: the average deoxyhaemoglobin responses to $(C)$ the chromatic gratings, and $(D)$ the moving gratings. The vertical bars (lower left) show the mean standard error (calculated over 1s) for the data in the figure. The horizontal grey bars indicate the period during which the stimulus was presented. 
For the chromatic gratings, repeated measures analyses of variance showed that there was an increase in the amplitude of the oxyhaemoglobin response with chromaticity separation $(F(2,30)=3.65, p=.038)$, the small colour difference producing a smaller response than the other chromaticity separations (Figure 3), a finding previously reported (Haigh et al., 2013). There was no significant difference in the amplitude of the PSU $(F(2,30)=2.50, p=.099)$; however, when the PSU (22s after stimulus-onset for a period of 10s) was subtracted from the amplitude of the response (6s after stimulus onset for a period of 10s), the small colour difference produced a smaller peak-to-trough difference than the other chromaticity separations $(F(2,30)=5.08, p=.013)$.

There was no significant effect of the grating on the slope of the offset of the oxyhaemoglobin response $(F(2,30)=0.98, p=.389)$, although the amplitude of the oxyhaemoglobin response was negatively correlated with the slope of the offset $(r(48)=-.67, p<.001)$ : larger responses had steeper offsets. The PSU was negatively correlated with slope of the offset $(r(48)=.41, p=.044)$ : steeper response slopes had larger PSUs.

There was a significant linear trend of chromaticity separation and steeper response onset $(F(1,15)=$ $5.66, p=.031$ ). This is likely due to the fact that the rising slope was positively correlated with the amplitude of the response $(r(48)=.75, p<.001$, and negatively correlated with the falling slope $(r(48)=-$ $.58, p<.001)$.
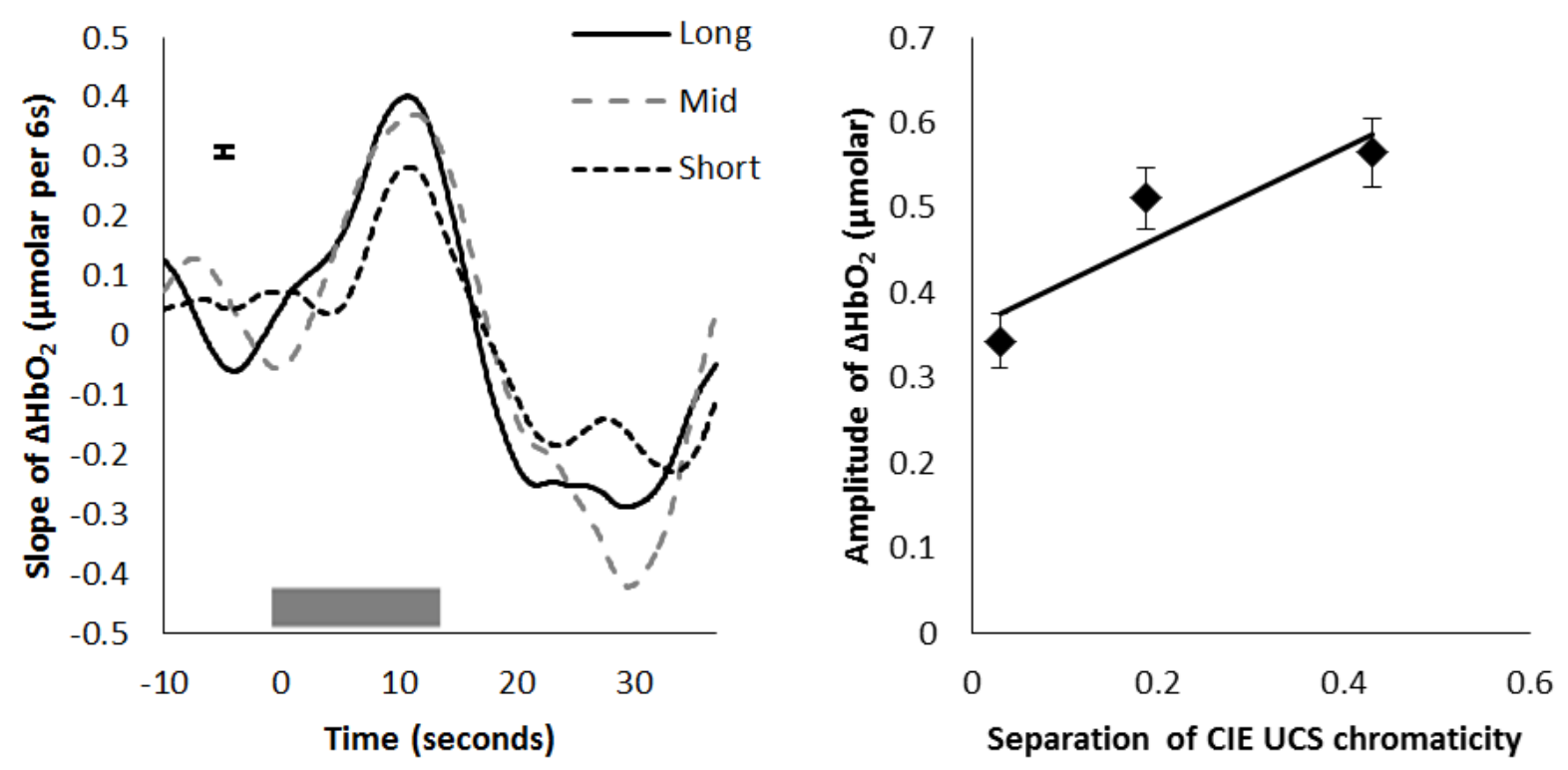
Figure 3. Left panel: slope of the haemodynamic response function shown in Figure 2, based upon the running average for the 6 s preceeding and following the point shown, as described in Data Analysis. The vertical bar shows the mean standard error (calculated over 1s) for the data in the figure. The horizontal grey bar indicates the period during which the stimulus was presented. Right panel: the amplitude of the oxyhaemoglobin response for each chromaticity separation; error bars show one standard error.

\subsection{Experiment 2}

For the moving gratings, there was no difference in the amplitude of the oxyhaemoglobin response between the responses to the three patterns $(F(2,34)=2.00, p=.150)$, but a difference in the slope of the response after stimulus-offset $(F(2,34)=3.29, p=.049)$, with linear contrast showing that the static gratings resulted in an average response in which the slope decreased from drifting to vibrating to static $(F(1,17)=7.301, p=.015)$. There was also a significant linear contrast in the amplitude of the PSU $(F(1,17)=2.41, p=.030)$, with the static grating producing the smallest PSU (Figure 4). The fall of the response was positively correlated with the size of the PSU $(r(54)=.69, p<.001)$ : the steeper responses had the largest PSUs. However, there was no significant difference in the peak-to-trough difference in the response to the three patterns $(F(2,34)=0.43, p=.654)$.
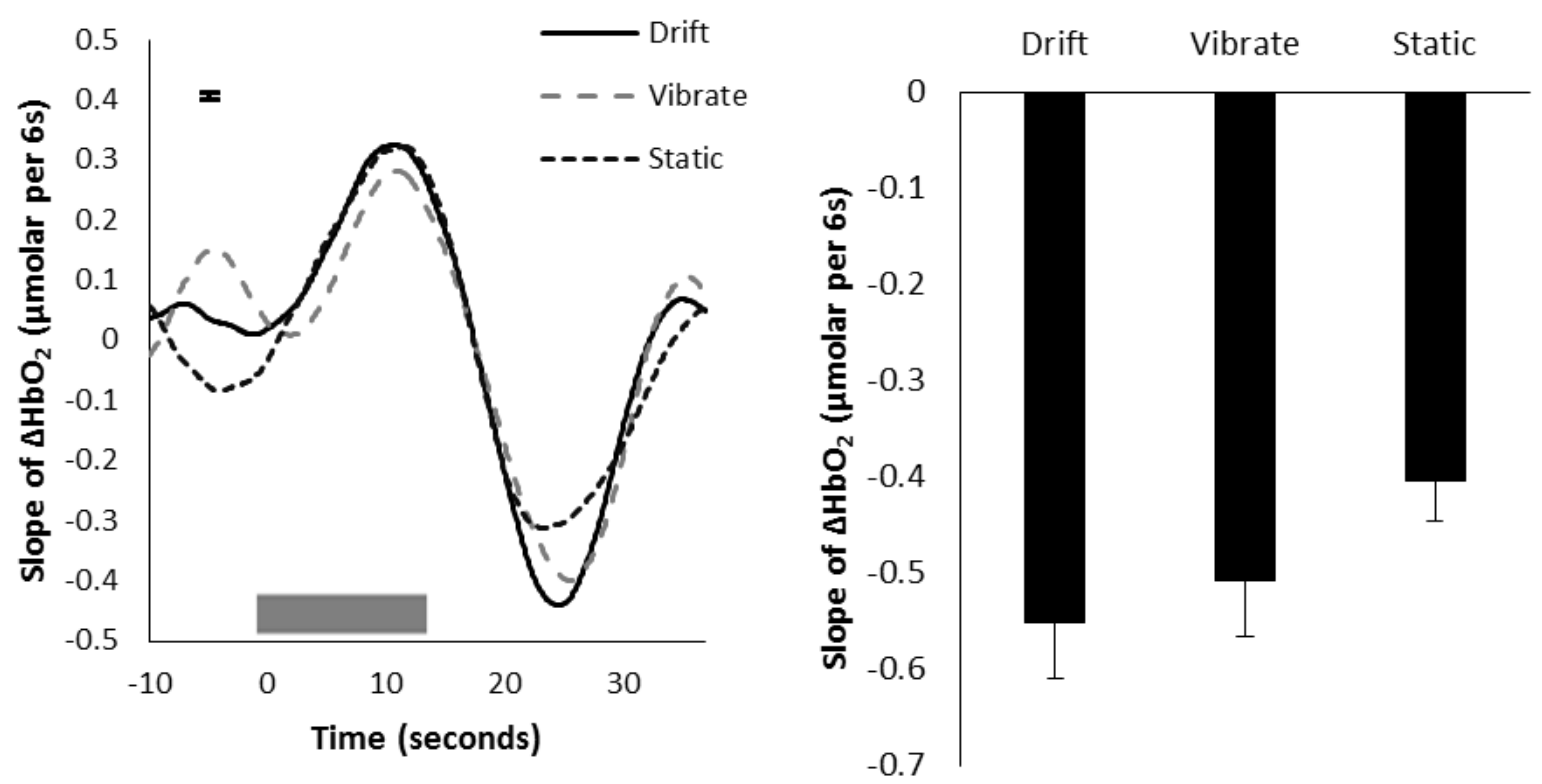

Figure 4. Left panel: slope of the haemodynamic response function shown in Figure 2 based upon the running average for the 6 s preceeding and following the point shown, as described in Data Analysis. The vertical bar shows the mean standard error (calculated over 1s) for the data in the figure. The horizontal 
grey bar indicates the period during which the stimulus was presented. Right panel: the slope of the oxyhaemoglobin response after stimulus offset from the drifting, vibrating and static gratings; error bars show one standard error.

Once again, the correlation between the amplitude and the slope of the offset of the oxyhaemoglobin responses from the achromatic gratings was significant $(r(54)=-.45, p<.001)$. There was no significant effect of the grating type on the rising slope $(F(2,34)=1.27, p=.293)$, but there was a positive correlation between the rising slope and the amplitude of the responses $(r(54)=.65, p<.001)$ and a negative correlation between the rising slope and the falling slope $(r(54)=-.46, p<.001)$. The rising slope may reflect individual differences in the oxyhaemoglobin response, but may not be sensitive to the stimulus itself.

To test the relationship between the amplitude and slope of the oxyhaemoglobin responses, the average amplitude and slope of the responses to the chromatic gratings (across chromaticity separation and colour-pair) was correlated with the average amplitude and slope of the responses to the moving gratings (across the drifting, vibrating and static patterns) for each individual. Sixteen participants had NIRS recordings that were used in both experiments. The amplitudes of the responses to the chromatic gratings was negatively correlated with the slope of the responses to the moving gratings $(r(14)=-.70$, $p=.002$ ). The amplitudes of the responses from the two experiments were positively correlated $(r(14)=.87, p<.001)$. This shows that individuals who produced a large haemodynamic response to the chromatic gratings were also those who produced the steeper response slopes to the moving gratings after stimulus-offset, suggesting that the neural mechanisms driving the large haemodynamic response amplitudes, were also those that were driving the steeper response offset.

To check that the differences in haemodynamic response to the gratings were not due to the method of analysis, the shape of the oxyhaemoglobin response was analysed using a least square regression of the response over time (from the response at stimulus-offset for 10s) calculated for each participant for each type of grating on both the smoothed and unsmoothed data. The amplitude of the responses was also recalculated using the unsmoothed data. The results were similar: for the response to the chromatic gratings, there was a significant difference in the amplitude of the oxyhaemoglobin response, but not in the shape; and for the response to the moving gratings, there was no significant difference in the amplitude of the response, but the drifting grating and vibrating grating produced a steeper slope after stimulus offset than the static grating. 


\section{Discussion}

The original aim of this study was to measure aspects of the haemodynamic response to stimuli that were known to vary in the discomfort they evoked and in their potential to induce seizures in patients with photosensitive epilepsy, the purpose being to characterize any associated differences in the nature of the haemodynamic response. For Experiment 1, the gratings with the greatest colour separation produced an oxyhaemoglobin response with the greatest amplitude, and this was also reflected in the post-stimulus undershoot (PSU) of the response after stimulus-offset. Gratings with large chromaticity separation have been reported to be perceived as uncomfortable to view, suggesting a relationship between discomfort and the amplitude of the cortical response (Haigh et al., 2013). However, for Experiment 2, there was no significant difference in the amplitude of the oxyhaemoglobin response between the drifting, vibrating and static gratings, even though the moving gratings were perceived as more uncomfortable to view than static (Haigh et al., 2012). The amplitudes to all three gratings were relatively high, possibly because the oxyhaemoglobin response saturated. Nevertheless, the shape of the oxyhaemoglobin response differed between the static, drifting and vibrating gratings. The moving gratings produced steeper slopes after stimulus-offset compared to the static gratings, and the poststimulus undershoot (PSU) was larger. The shape of the oxyhaemoglobin response did not differ at stimulus-onset (at the rise of the response). The rising slope appears to be related to the amplitude of the oxyhaemoglobin response, and did not consistently distinguish between the parameters of the stimuli. For Experiment 2, the similarity of the amplitudes reduced its sensitivity to the grating motion. The falling slope, however, differed depending on the stimulus motion.

Differences in the shape of the haemodynamic response have been reported previously, in relation to local GABA concentrations (Muthukumaraswamy et al., 2012) and cortical hyper-excitability (such as in migraineurs) (Coutts et al., 2012). We show that the shape of the haemodynamic response can differ consistently from one stimulus to another. Furthermore, the shape of the haemodynamic response appears to reflect the strength of the stimulus - stimuli that evoke the most discomfort and are potentially epileptogenic, produce the largest amplitude and the steepest slope in the oxyhaemoglobin response. A direct comparison of haemodynamic responses with measures of discomfort in the same observers would strengthen this claim.

In the current study, we have shown that the stimulus can affect the amplitude or the slope of the response. However, it must be noted that the amplitude and the slope of the response were correlated in response to the stimuli presented in Experiment 1 and in Experiment 2 such that the larger responses 
generally had a steeper offset. In addition, the participants who produced the largest oxyhaemoglobin responses in Experiment 1 to the chromatic gratings, also produced the steepest slope in their responses in Experiment 2 to the moving gratings. It is logical that larger response amplitudes would result in steeper offsets, but there was no significant difference in the amplitude of the responses to the moving gratings, even though there was a significant difference in the slope of the response. This suggests that the slope of the response can be associated with the extent of the cortical activation independently of the amplitude of the response.

Both the amplitude of the response and the slope of the response appear to influence the PSU of the haemodynamic response, which occurs after stimulus-offset before the response returns to baseline (Davis et al., 1994; Savoy et al., 1995). The PSU has been measured more extensively in the BOLD response, although has also been found in the NIRS haemodynamic response previously (Huppert et al., 2006). The duration and the magnitude of the PSU increases with stimulus strength (varying stimulus duration and contrast) (Vazquez \& Noll, 1998). There is little between-subject variation in the duration of the PSU to the same stimulus, but there is spatial variability in its magnitude (Mildner et al., 2001; Yacoub et al., 2006), potentially similar to the individual differences in the shape of the haemodynamic response reported previously (Coutts et al., 2012; Muthukumaraswamy et al., 2012), consistent with differences in cortical excitability.

The mechanisms behind the PSU have been heavily debated. It is possible that the PSU is due to vascular recovery and is not neural in origin. There is increasing evidence to suggest that the PSU can be attributed to temporal mismatches between cerebral blood flow and cerebral blood volume or cerebral oxygenation consumption (Frahm et al., 1996; Silva, Koretsky \& Duyn, 2007), with a review paper concluding that the PSU is due to increased sustained oxygen metabolism when cerebral blood volume and blood flow has returned to baseline (van Ziji, Hua \& Lu, 2012). However, the individual differences in PSU magnitude could be related to inhibitory mechanisms. Muthukumaraswamy et al. (2012) report that the shape of the BOLD response was correlated with local GABA-concentration: the taller BOLD responses were negatively correlated with GABA concentration, and the wider BOLD responses were positively correlated with GABA concentration. Logothetis et al. (2001) also noted that the poststimulus undershoot was preceded by inhibition in the neural response. It is possible that changes in inhibitory mechanisms also play a role in the shape of the haemodynamic response post-stimulus. 


\section{Acknowledgements}

We would like to thank Roger Deeble and Roger Grace for their technical help. This work was partly funded by the Wellcome Trust (Grant \#: 080274 to AJW).

\section{References}

Aurora, S. K., \& Wilkinson, F. (2007). The brain is hyperexcitable in migraine. Cephalalgia, 27(12), 14421453.

Binnie, C. C., Findlay, J., \& Wilkins, A, J. (1985). Mechanisms of epileptogenesis in photosensitive epilepsy implied by the effects of moving patterns.pdf. Electroencephalography and Clinical Neurophysiology, 61, 1-6.

Brainard, D. H. (1997). The Psychophysics Toolbox. Spatial Vision, 10(4), 433-436.

Coutts, L. V, Cooper, C. E., Elwell, C. E., \& Wilkins, A. J. (2012). Time course of the haemodynamic response to visual stimulation in migraine, measured using near-infrared spectroscopy. Cephalalgia, 32 (8), 621-629.

Davis, T. L., Weisskoff, R. M., Kwong, K. K., Savoy, R., \& Rosen, B. R. (1994). Susceptibility contrast undershoot is not matched by inflow contrast undershoot. In Proceedings of the SMR 2nd Annual Meeting, San Francisco (p. 435).

Duncan, A. et al. (1996). Measurement of cranial optical path length as a function of age using phase resolved near infrared spectroscopy. Pediatric Research, 39(5), 889-894.

Frahm, J., Krüger, G., Merboldt, K.-D., \& Kleinschmidt, A. (1996). Dynamic uncoupling and recoupling of perfusion and oxidative metabolism during focal brain activation in man. Magnetic Resonance in Medicine, 35(2), 143-148.

Gallagher, A., Lassonde, M., Bastien, D., Vannasing, P., Lesage, F., Grova, C., ... Nguyen, D. K. (2008). Noninvasive pre-surgical investigation of a 10 year-old epileptic boy using simultaneous EEG-NIRS. Seizure, 17(6), 576-582.

Haigh, S. M., Barningham, L., Berntsen, M., Coutts, L. V, Hobbs, E. S. T., Irabor, J., ... Wilkins, A. J. (2013). Discomfort and the cortical haemodynamic response to coloured gratings. Vision Research, 89(0), 47-53.

Haigh, S. M., Cooper, N., Romei, V. \& Wilkins, A. J. (2013). Alpha, gamma and the haemodynamic responses - how are they related? Perception, 42 (ECVP Abstract Supplement), 236.

Haigh, S. M., Karanovic, O., Wilkinson, F., \& Wilkins, A. J. (2012). Cortical hyperexcitability in migraine and aversion to patterns. Cephalalgia , 32 (3), 236-240. 
Huang, J., Cooper, T. G., Satana, B., Kaufman, D. I., \& Cao, Y. (2003). Visual Distortion Provoked by a Stimulus in Migraine Associated With Hyperneuronal Activity. Headache: The Journal of Head and Face Pain, 43(6), 664-671.

Huppert, T. J., Hoge, R. D., Diamond, S. G., Franceschini, M. a, \& Boas, D. a. (2006). A temporal comparison of BOLD, ASL, and NIRS hemodynamic responses to motor stimuli in adult humans. Neurolmage, 29(2), 368-82.

Kleiner, M., Brainard, D., Pelli, D., Ingling, A., Murray, R., \& Broussard, C. (2007). What 2 new in Psychtoolbox-3. Perception, 36(14), 1.

Klimesch, W., Sauseng, P., \& Hanslmayr, S. (2007). EEG alpha oscillations: The inhibition-timing hypothesis. Brain Research Reviews, 53(1), 63-88.

Lareau, E., Lesage, F., Pouliot, P., Nguyen, D., Le Lan, J., \& Sawan, M. (2011). Multichannel wearable system dedicated for simultaneous electroencephalography/near-infrared spectroscopy real-time data acquisitions. Journal of Biomedical Optics, 16(9), 96014.

Logothetis, N. K., Pauls, J., Augath, M., Trinath, T., \& Oeltermann, A. (2001). Neurophysiological investigation of the basis of the fMRI signal. Nature, 412(6843), 150-157.

Mildner, T., Norris, D. G., Schwarzbauer, C., \& Wiggins, C. J. (2001). A qualitative test of the balloon model for BOLD-based MR signal changes at 3T. Magnetic Resonance in Medicine, 46(5), 891-899.

Moosmann, M., Ritter, P., Krastel, I., Brink, A., Thees, S., Blankenburg, F., ... Villringer, A. (2003). Correlates of alpha rhythm in functional magnetic resonance imaging and near infrared spectroscopy. Neurolmage, 20(1), 145-158.

Muthukumaraswamy, S. D., Evans, C. J., Edden, R. a E., Wise, R. G., \& Singh, K. D. (2012). Individual variability in the shape and amplitude of the BOLD-HRF correlates with endogenous GABAergic inhibition. Human Brain Mapping, 33(2), 455-65.

Olman, C. A., Ugurbil, K., Schrater, P., \& Kersten, D. (2004). BOLD fMRI and psychophysical measurements of contrast response to broadband images. Vision Research, 44(7), 669-683.

Parra, J., Lopes da Silva, F. H., Stroink, H., \& Kalitzin, S. (2007). Is colour modulation an independent factor in human visual photosensitivity? Brain : A Journal of Neurology, 130(Pt 6), 1679-89.

Pelli, D. G. (1997, January 1). The VideoToolbox software for visual psychophysics: transforming numbers into movies. Spatial Vision.

Roche-Labarbe, N., Zaaimi, B., Berquin, P., Nehlig, A., Grebe, R., \& Wallois, F. (2008). NIRS-measured oxy- and deoxyhemoglobin changes associated with EEG spike-and-wave discharges in children. Epilepsia, 49(11), 1871-1880.

Rovati, L., Salvatori, G., Bulf, L., \& Fonda, S. (2007). Optical and electrical recording of neural activity evoked by graded contrast visual stimulus. Biomedical Engineering Online, 6, 28. 
Savoy, R. L., Bandettini, P. A., O'Craven, K. M., Kwong, K. K., Davis, T. L., Baker, J. R., ... Rosen, B. R. (1995). Pushing the temporal resolution of fMRI: Studies of very brief visual stimuli, onset variability and asynchrony, and stimulus-correlated changes in noise. In Program of SMR presented at 3d Annual Meeting, Nice, France.

Schadow, J., Lenz, D., Thaerig, S., Busch, N. A., Fründ, I., Rieger, J. W., \& Herrmann, C. S. (2007). Stimulus intensity affects early sensory processing: Visual contrast modulates evoked gamma-band activity in human EEG. International Journal of Psychophysiology, 66(1), 28-36.

Semyanov, A., Walker, M. C., \& Kullmann, D. M. (2003). GABA uptake regulates cortical excitability via cell type--specific tonic inhibition. Nature Neuroscience, 6(5), 485.

Silva, A. C., Koretsky, A. P., \& Duyn, J. H. (2007). Functional MRI impulse response for BOLD and CBV contrast in rat somatosensory cortex. Magnetic Resonance in Medicine, 57(6), 1110-1118.

Swettenham, J. B., Muthukumaraswamy, S. D., \& Singh, K. D. (2013). BOLD Responses in Human Primary Visual Cortex are Insensitive to Substantial Changes in Neural Activity. Frontiers in Human Neuroscience, 7 (March), 76.

Tong, Y. et al. (2005). Studying brain function with near-infrared spectroscopy concurrently with electroencephalography. Proc. SPIE.

Van Zijl, P. C. M., Hua, J., \& Lu, H. (2012). The BOLD post-stimulus undershoot, one of the most debated issues in fMRI. Neurolmage, 62(2), 1092-1102.

Vazquez, A. L., \& Noll, D. C. (1998). Nonlinear Aspects of the BOLD Response in Functional MRI. Neurolmage, $7(2), 108-118$.

Villringer, A., Planck, J., Hock, C., Schleinkofer, L., \& Dirnagl, U. (1993). Near infrared spectroscopy (NIRS): A new tool to study hemodynamic changes during activation of brain function in human adults. Neuroscience Letters, 154(1-2), 101-104.

Yacoub, E., Ugurbil, K., \& Harel, N. (2006). The spatial dependence of the poststimulus undershoot as revealed by high-resolution BOLD- and CBV-weighted AMRI. Journal of Cerebral Blood Flow and Metabolism : Official Journal of the International Society of Cerebral Blood Flow and Metabolism, 26(5), 634-644. 